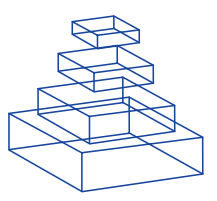

\title{
Circuit neuroscience: the road ahead
}

\author{
Rafael Yuste \\ HHMI, Department of Biological Sciences, Columbia University, USA \\ Correspondence: rmy5@columbia.edu
}

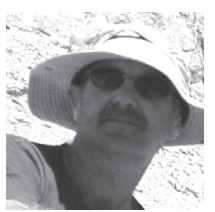

Rafael Yuste studied Medicine at the Universidad Autonoma and the Fundacion Jimenez Diaz Hospital in Madrid. After a brief period in Sydney Brenner's group at the LMB in Cambridge, he obtained his $P h D$ with Larry Katz in Torsten Wiesel's laboratory, at Rockefeller University in New York. He then moved to Bell Labs, where he was a postdoctoral student of David Tank and also worked with Winfried Denk. In 1996 he joined the Department of Biological Sciences at Columbia University, where he is currently Tenured Professor. In 2005 he became HHMI Investigator and co-director of the Kavli Institute for Brain Circuits at Columbia. Since 1997 he is visiting researcher in Javier DeFelipe's laboratory at the Cajal Institute in Madrid. Yuste's laboratory studies the structure and function of cortical circuits and the biophysical properties of dendritic spines.
It is difficult to write about grand challenges in our field without pontificating or pretending to show a degree of certainty in assessing the field that I do not possess. I would rather comment on a few of the issues that particularly worry me. Therefore, this article is just a snapshot of our field now, as I see it, and encourage readers to read it as the opinion of just one of their colleagues.

My comments are aimed at Circuit Neuroscience. What exactly is Circuit Neuroscience? As stated in the mission statement of Frontiers in Neural Circuits, I follow the definition of Circuit Neuroscience as the understanding of the computational function of neural circuits, linking this function with the circuit micro-structure. Within this field, I will address three different types of challenges: scientific, methodological and sociological ones.

\section{Scientific problems:}

I think that it is fair to say that we are profoundly ignorant about the structure and function of neural circuits. One could say that the goal of our field is to reverse-engineer biological circuits and that in order to do so we need to know their structure and logic, so that we can understand their computational algorithms. Like engineering students in their final college exam, we are attempting to decipher the "transfer function" of unknown circuits, this time biological ones. To do so effectively, for most neural circuits in most species, we need to solve the following problems:

Cell Types Problem: In terms of the structure of neural circuits, one of the fundamental problems we are facing generally is that we still do not know which is the exact complement of neuronal cell types present. In spite of more than a hundred years of neuroanatomy, this issue is still not resolved and it is difficult to imagine how we could reverse engineer a circuit without knowing the list of its parts. Why are we still ignorant of this list? Besides the fact that the neuronal cell types don't come pre-labeled and that they are normally mixed together, anatomical efforts in the past have been essentially qualitative, often without clear criteria to differentiate between cell types. The introduction of quantitative anatomical approaches is greatly helping in discerning among cell types. Also, classifications of neurons are increasingly relying on a multifaceted description of their phenotypes, encompassing not just anatomical, but also electrophysiological and molecular 
features. Particularly powerful is the generation of transgenic animals were neuronal cell types can be actually pre-labeled genetically. A next logical step would involve the systematic use of multivariate statistics with which to explore this multidimensional space and define most, or all, the cell types present. Towards this goal, common efforts by many laboratories, such as the recent Petilla interneuron nomenclature meeting, could standardize the nomenclature and, together with the new more comprehensive type of data on each neuron type (anatomical, physiological and molecular) and their standardization in databases, lead to a universally agreed parts list of most regions of the CNS.

Circuit Connectivity Problem: Another fundamental challenge is to decipher the connectivity diagram of neural circuits, one of the holy grails of Neuroscience. Again, in spite of a century of work, we are still at the beginning of this formidable task. With few exceptions, most brain regions are still the "impenetrable jungles where many investigators have lost themselves" that Cajal wrote about. As discussed below, there is hope that in the near future, technical advances will break open this problem, allowing the description, for the first time, of the basic synaptic microcircuits of at least some regions of the brain. Together with the "cell type" problem, this "circuit connectivity" problem is perhaps the biggest Neuroscience breakthrough that could be solved within our lifetimes, revealing the actual structure of neural circuits. What the genome project was for Molecular Biology, the Circuit Connectivity project could be for Neuroscience. This project could help galvanize public opinion and catalyze funding, and it represents a unique opportunity for younger generations of researchers.

Circuit Algorithm Problem. As in Engineering, knowledge of the circuit diagram is just one step, albeit a necessary one, to understand the logic and computational algorithms that are implemented in the circuit. I am afraid that we are completely ignorant of such logic for essentially all neuronal circuits. While this is a sad state of affairs, it is at the same time, virgin territory for future efforts, and for this reason, it is difficult to imagine a more exciting time to work in this field. In this respect, a fundamental problem is to discern general strategies when comparing circuits from different parts of the CNS, or from different species. There is practically zero effort nowadays in comparing circuits, yet is seems that this should be essential not only to provide perspective on any one circuit, but also to help the advances by realizing that similar strategies could be used by different circuits. Although perhaps this could be disregarded as 19th century "armchair science", the close comparison of the similarities and differences in structure and algorithms among species and parts of the brain appears necessary and could lead to powerful insights.

Circuit Dynamics Problem. A related issue is the better understanding of the temporal dynamics of biological circuits. There have been many meritorious efforts to figure out this logic or "transfer function", by approaching the function of the neural circuits from the systems level, treating them essentially as a black box. These approaches could yield fundamental insights into the algorithms used by neural circuits, particularly if their function can be described by the "Sherringtonian" hypothesis that they serve to transform sensory inputs into motor outputs. On the other hand, it is becoming clear that neural circuits in all species have a very rich "inner life", manifested by a high degree of organized spontaneous activity. In some cases, this intrinsic activity not only interacts meaningfully with the sensory inputs, but also can even generate behavior in the absence of sensory stimuli. In this "Kantian" view of the mind, the brain is essentially interested in its own activity. If this is indeed the case, we will not be able to understand how it interact with sensory information to generate behavior, until we enter into these circuits and decipher their inner lives. Thus, it becomes essential to capture the dynamical activity of neural circuits in their entirety. Neurophysiologists have traditionally recorded from individual neurons and only recently, particularly through the development of novel methods (see below), we are starting to visualize and comprehend the larger scale dynamics of neuronal circuits. This information is not a minor detail to be added to the database, but essential to understand the computational strategies employed by neural circuits, since the dynamical nature of the activity can help pinpoint quite precisely what types of circuit models could be operating, out of an infinite number of potential algorithms. This area of work is one of the most exciting ones in modern Neuroscience and has yielded tantalizing pieces of data highlighting the temporal precision and non-random structure of the spontaneous and evoked neural activity. While these results are not 
universally accepted, it is fair to say that they are already challenging our most fundamental conceptions about how neural information is coded and transmitted.

\section{Methodological problems:}

As in many areas of Science, new methods drive research in Neuroscience and are normally responsible for the introduction of novel paradigms and ideas. In circuit neuroscience in particular, the need for novel techniques is particularly acute. More specifically, there are four significant technical challenges ahead.

Monitoring the Activity of Neuronal Ensembles. The use of optical tools appears to be here to stay. Imaging techniques, in spite of their relative novelty, are already responsible for a significant push ahead of many areas of circuit neuroscience. I personally have felt that recent imaging meetings, such as the one organized at Cold Spring Harbor on alternate years, are among the most exciting ones I have ever attended. The reason for this is that one can visualize, for the first time, the activity of neuronal ensembles, and it is possible that the function of CNS circuits is organized at this emergent level. Like trying to read a newspaper with a microscope, recording from single cells may not allow us to discover what neural circuits are doing. Only after we take a step back and visualize the joint activity of hundreds, or perhaps thousands of neurons, we may encounter the units that are the framework on which the function of the brain is built. Currently, calcium imaging appears to be the preferred technique to perform these population measurements, even though it is curious how this technique was viewed with great suspicion only a few years ago. Even today, it is still not widely exploited, even for problems where it could yield significant advances. Nevertheless, having been involved in the development of calcium imaging of circuits, I can be particularly critical of it and point out that we still need better methods that would allow us to image voltage in neuronal circuits with single cell resolution. To me, this the single most important technical challenge in Neuroscience. Novel forms of microscopy, such as second harmonic generation, or the introduction of novel molecular sensors of voltage, could revolutionize the study of neural circuits.

Manipulating Neuronal Activity. Hand in hand with novel imaging techniques are approaches to optically manipulate individual neurons or populations of them, by either activating or inactivating them. This is the engineers' favorite tool in their attempts to decipher transfer functions: to be able to test any circuit component at will. Besides photo-uncaging, the influx of molecular tools have changed the landscape of neuronal activity manipulations. While current techniques are extremely useful, they also have some technical problems, so the development of novel methods to perform these experiments and investigate these questions still remains a significant challenge.

Visualizing Synaptic Connections. Another very exciting area of current research is the introduction of novel methods that allow the systematic determination of synaptic connectivity. There is an embarrassment of riches right now, from novel ultra-structural methods, to the use of glutamate photo-stimulation, viral approaches and a host of genetically based strategies, among others. Any one of these techniques could be in principle capable to be the one that could lead us to the "promised land". The challenge is to further refine these techniques so that they actually yield what they promise, and of course encourage the development of novel ones.

Computational and Theoretical Methods. A fundamental problem that I feel Circuit Neuroscience is encountering is that we are essentially lacking a general theory of neural circuits. Experimentalists are entering a territory without a map and without basic theoretical tools and even a vocabulary with which to even be able to ask the right questions. With some notable exceptions, the impact of theory in Neuroscience has traditionally been weak, yet in recent decades a stream ideas coming from Physics, Mathematics and Computer science are becoming more and more influential. For example, concepts such as dynamical systems, circuit attractors, information theory, Bayesian coding and temporal multiplexing are now driving experimental programs in many laboratories in Circuit Neuroscience. I think that these theoretical approaches are essential as we move into the future. At the same time, it is likely that we still have not developed the correct mathematical formalisms with which to decipher the multi-neuronal dynamics we can already observe. 


\section{Sociological problems: our publication culture}

I want to finish on a personal note, on what I consider may be one of the biggest challenges to the advancement of circuit neuroscience. This is not a scientific or technical challenge but one that relates to the fundamental way in which we scientists organize ourselves as a community. I think that the current publication system is deeply flawed and constitutes a major obstacle in our research today. This reflection is based in my own experience of watching the reviewing system disintegrate during the last twenty years. The system of anonymous peer-reviewing of manuscripts, while it might have worked well before, is pretty much ineffective today. This could be due perhaps to the massification and industrialization of the research enterprise, the system of career advancements based on publishing papers in selective journals, and the degradation of the quality and criteria by which articles are reviewed and by which journal editors decide to accept or reject them. But regardless of the reason, in my own experience I have unfortunately observed that the zero-sum game, where the contributions of other laboratories than one's own are viewed negatively, is prevalent. I suspect that the anonymity and lack of accountability of the review process has led to its corruption. It seems to me that the purpose of most reviewing nowadays is to block the publication of the work, or steer the direction of the research, rather than actually help the authors improve their science. As pointed out by social scientists and economists before, anonymity does not encourage the most altruistic instincts in humans. As a consequence of this, it is not uncommon to spend more time and effort in the publication process than in the actual generation and analysis of the scientific data.

Because publication is the only output of scientific research, this is a very serious problem, with negative repercussions for the entire scientific process. In fact, one of the worse consequences of this essentially negative system of reviewing and publishing is that younger researchers can become disheartened with the system and abandon the field, or abandon science altogether. Unfortunately, I have experienced this closely, having seen bright promising students been trounced upon by negative reviewers time after time, eventually deciding to drop out. Instead, these students should have been encouraged, rewarded and lauded by their peers, as our ticket to the future. Because circuit neuroscience is a nascent discipline, I find this situation extremely dangerous for our particular field, whose success depends critically on the ability to attract the best young minds.

Our biggest challenge is therefore to fundamentally change the methods by which we review our papers, publish our work and, importantly, allocate credit and reward careers, since changes in the publication system will be moot without similar changes in the reward system. Precisely for this reason I am embracing the Frontiers enterprise, and I encourage readers to join in. I am optimistic, and I think that as a community we can recover the spirit of excitement of the scientific research, which presumably attracted all of us to devote our lives to science. As in the past, we should be able to open the journals with excitement, genuinely looking forward to learn about novel results, discoveries and advances, rather than with trepidation of our competition succeeding and our own standing potentially decreasing and thus jeopardizing our future. Science is very exciting and should be fun. As students of neural circuits, we are privileged to work on one of the fundamental problems in the history of science, deciphering the function of the brain. We have a duty to help mankind and advance its knowledge of this key problem, one that will have enormous consequences for many aspects of human life, society and medicine. We have a phenomenal task ahead of us. For these reasons, we should work as a team of intelligent collaborators who use their skills to help each other advance further, knowing that the success of the entire enterprise is much more important that the role of each individual.

Front. Neurosci. (2008) 2, 1: 6-9. doi: 10.3389/neuro.01.017.2008

Copyright $\odot 2008$ Yuste. This is an open-access publication subject to an exclusive license agreement between the authors and the Frontiers Research Foundation, which permits unrestricted use, distribution, and reproduction in any medium, provided the original authors and source are credited. 